\title{
Duality theorem for topological semigroups
}

\author{
Nobuhiko Tatsuuma
}

\begin{abstract}
For topological semigroups $S$, we consider Tannaka-type duality theorems, which are extensions of the notion of weak Tannaka duality theorem for topological groups. In the case of topological semigroups, we must set as the dual object of $S$ all isometric representations of $S$ instead of all unitary representations. We define a property $T$-type for $S$. After arguments analogous to previous work from the author, we can prove that our Tannaka-type duality theorem is valid if and only if $S$ is a T-type semigroup.
\end{abstract}

\section{Tannaka-type duality theorem}

A topological semigroup $S$ is a semigroup with unit $e$ which is simultaneously a topological space and whose semigroup operation is continuous. An isometric representation of $S$ is a continuous homomorphism from $g \in S$ to the semigroup $\left\{T_{g}\right\}$ of isometric operators on a Hilbert space $\mathcal{H}$ with weak topology. In this paper, hereafter we call an isometric representation simply a representation.

For an isometric operator $J$ on $\mathcal{H}$ and $\forall c \in \mathbf{C}, \forall v, u, v \perp u \in \mathcal{H}$,

$$
\begin{aligned}
\|v\|^{2}+|c|^{2}\|u\|^{2} & =\|v+c u\|^{2}=\|J(v+c u)\|^{2} \\
& =\|J v\|^{2}+|c|^{2}\|J u\|^{2}+2 \Re(\bar{c}\langle J v, J u\rangle) \\
& =\|v\|^{2}+|c|^{2}\|u\|^{2}+2 \Re(\bar{c}\langle J v, J u\rangle),
\end{aligned}
$$

where $\Re$ shows the real part. This implies that $(J v, J u)=0$ and $J v \perp J u$. Hence, for an orthonormal system $\left\{v_{\alpha}\right\},\left\{J v_{\alpha}\right\}$ gives an orthonormal system too.

Let $\mathcal{H}^{1}$ and $\mathcal{H}^{2}$ be Hilbert spaces, and take complete orthonormal systems $\left\{v_{\alpha}^{1}\right\}$ in $\mathcal{H}^{1}$ and $\left\{v_{\alpha}^{2}\right\}$ in $\mathcal{H}^{2} ;\left\{v_{\alpha}^{1} \otimes v_{\beta}^{2}\right\}$ is an orthonormal system in $\mathcal{H}^{1} \otimes \mathcal{H}^{2}$. Therefore we can define the tensor product $J^{1} \otimes J^{2}$ for any isometric operators $J^{k}$ on $\mathcal{H}^{k}(k=1,2)$ as an isometric operator in $\mathcal{H}^{1} \otimes \mathcal{H}^{2}$.

Let $\Omega \equiv\left\{D=\left(\mathcal{H}^{D}, T_{g}^{D}\right)\right\}$ be the set of all representations of a given topological semigroup $S$ whose dimensions are bounded by $\max \left(\aleph_{0}, \# S\right)$, and consider operations between elements of $\Omega$ as

(1) unitary equivalence: $D_{1} \sim_{W} D_{2}$ (W: intertwining unitary operator),

(2) subrepresentation: $D_{1} \succ D_{2}$,

Kyoto Journal of Mathematics, Vol. 55, No. 3 (2015), 543-554

DOI 10.1215/21562261-3089046, (C) 2015 by Kyoto University

Received September 18, 2012. Revised January 21, 2014. Accepted June 3, 2014.

2010 Mathematics Subject Classification: 22A25, 22D35. 
(3) tensor product: $D_{1} \otimes D_{2}$,

(4) contragradient representation: $D \rightarrow \bar{D}$.

Consider an operator field $\mathbf{A} \equiv\left\{A^{D}\right\}_{D \in \Omega}$ on $\Omega$ satisfying

(Cd-0) for each $D \in \Omega, A^{D}$ is an isometric operator on the representation space $\mathcal{H}^{D}$

(Cd-1) $D_{1} \sim_{W} D_{2} \Rightarrow W A^{D_{1}} W^{-1}=A^{D_{2}}$,

(Cd-2) $\left.D_{1} \succ D_{2} \Rightarrow A^{D_{1}}\right|_{\mathcal{H}^{D_{2}}}=A^{D_{2}}$

(Cd-3) $A^{D_{1}} \otimes A^{D_{2}}=A^{D_{1} \otimes D_{2}}$,

(Cd-4) $\overline{A^{D}}=A^{\bar{D}}$.

We call such an operator field $\mathbf{A}$ a birepresentation of $S$, and we write $\mathcal{J}$ for the set of all birepresentations. On the space $\mathcal{J}$, induce a topology, the product of weak topologies $\tau^{D}(D \in \Omega)$ on each component operator space on the Hilbert space $\mathcal{H}^{D}$. It is easy to see that, for any two birepresentations $\mathbf{A}_{j} \equiv\left\{A_{j}^{D}\right\}(j=$ $1,2)$, their product $\mathbf{A}_{1} \mathbf{A}_{2} \equiv\left\{A_{1}^{D} A_{2}^{D}\right\}$ is also a birepresentation. This product operation is continuous with respect to the above topology. So $\mathcal{J}$ is a topological semigroup.

Obviously for any $g \in S$ the operator field $\mathbf{T}_{g} \equiv\left\{T_{g}^{D}\right\}_{D \in \Omega}$ gives a birepresentation. Our weak Tannaka-type duality theorems assert the converses.

\section{ASSERTION}

For any birepresentation $\mathbf{A} \equiv\left\{A^{D}\right\}_{D \in \Omega}$, there exists a unique $g \in S$ such that $A^{D}=T_{g}^{D}(\forall D \in \Omega)$. Moreover, the topology on $\mathcal{J}$ given above coincides with the original topology of $S$ under the correspondence $g \mapsto \mathbf{T}_{g}$.

\section{Separating system of isometric representations, completeness, and T-type semigroups}

Hereafter, $S$ is a Hausdorff (i.e., $T_{2}$-)topological semigroup.

\section{DEFINITION 2.1}

A set $\Omega_{0} \equiv\left\{D_{\alpha} \equiv\left\{\mathcal{H}^{D_{\alpha}}, T_{g}^{D_{\alpha}}, v^{D_{\alpha}}\right\} \mid v^{D_{\alpha}}\right.$ is a normalized cyclic vector, $\left.\alpha \in A\right\}$ of cyclic isometric representations of $S$ gives a separating system of isometric representations (SSIR) if and only if, for any neighborhood $V$ of any element $g_{0}$ in $S$, there exist $D \in \Omega_{0}$ and $\varepsilon>0$ such that

$$
F\left(D, \varepsilon, g_{0}\right) \equiv\left\{g \in S|| 1-\left\langle T_{g}^{D} v^{D}, T_{g_{0}}^{D} v^{D}\right\rangle \mid<\varepsilon\right\} \subset V .
$$

We denote by $\mathbf{J}(\mathcal{H})$ the space of all isometric operators on a Hilbert space $\mathcal{H}$ and introduce the weak topology on it.

For any $J_{0}, J \in \mathbf{J}(\mathcal{H})$,

$$
\left\|J v-J_{0} v\right\|^{2}=-2 \Re\left(\left\langle J v-J_{0} v, J_{0} v\right\rangle\right) .
$$

$\dagger$ For definitions and some properties of contragradient representations, we refer the reader to $[4$, Section 1$]$. 
So, on $\mathbf{J}(\mathcal{H})$ the weak topology coincides with the strong topology. Moreover, $\mathbf{J}(\mathcal{H})$ becomes a topological semigroup with the multiplication of operators and this topology.

Let $D \equiv\left\{\mathcal{H}^{D}, T_{g}^{D}\right\}$ be any representation of $S$. The map $S \ni g \mapsto T_{g}^{D} \in$ $\mathbf{J}\left(\mathcal{H}^{D}\right)$ is continuous for each $D$, by definition.

Construct $\mathbf{J}(\Omega) \equiv \prod_{D \in \Omega} \mathbf{J}\left(\mathcal{H}^{D}\right)$ with the natural product topology. The map

$$
S \ni g \mapsto\left(T_{g}^{D}\right)_{D \in \Omega} \in \mathcal{J} \subset \prod_{D \in \Omega} \mathbf{J}\left(\mathcal{H}^{D}\right)=\mathbf{J}(\Omega)
$$

is into-homomorphisms as topological semigroups.

Write $S_{J}$ as the image of $S$ in $\mathbf{J}(\Omega)$. The existence of an SSIR for a $T_{2^{-}}$ topological semigroup $S$ shows that

(a) the map (2.3) is a one-to-one map from $S$ to $S_{J}$,

(b) the inverse map of (2.3) from $S_{J}$ (with restricted topology from $\mathbf{J}(\Omega)$ ) to $S$ is continuous.

So $S$ is embedded as a topological semigroup in $\mathbf{J}(\Omega)$. The following lemma is then obvious.

\section{LEMMA 2.1}

Let $S$ be a $T_{2}$-topological semigroup with an SSIR. Our weak Tannaka-type duality theorem is equivalent to $S_{J}=\mathcal{J}$ and the map (2.3) being an isomorphism between $S$ and its image $S_{J}=\mathcal{J}$ as topological semigroups.

On a $T_{2}$-topological semigroup $S$ with an SSIR $\Omega_{0}$, put

$$
\mathcal{W} \equiv\left\{W(D, \varepsilon) \equiv\left\{\left(g_{1}, g_{2}\right) \in S \times S \mid\left\|T_{g_{1}}^{D} v^{D}-T_{g_{2}}^{D} v^{D}\right\|<\varepsilon\right\}\left(D \in \Omega_{0}, \varepsilon>0\right)\right\} .
$$

It is easy to see that $\mathcal{W}$ gives a fundamental system of entourages on $S \times S$, and defines a uniform structure on $S$ (see [1]).

\section{DEFINITION 2.2}

A filter base $\mathcal{F} \equiv\left\{F_{\alpha}\right\}_{\alpha \in \Gamma}$ (where $\Gamma$ is a partially ordered set) on $S$ is called Cauchy if, for any entourage $W(D, \varepsilon)$, there exists an $\alpha \in \Gamma$ such that

$$
\forall \beta \succ \alpha, \quad \forall g_{1}, g_{2} \in F_{\beta}, \quad\left(g_{1}, g_{2}\right) \in W(D, \varepsilon) .
$$

We consider the topological semigroup $\mathbf{S} \equiv \mathbf{J}(\Omega)=\prod_{D \in \Omega} \mathbf{J}\left(\mathcal{H}^{D}\right)$. The identical representation of $\mathbf{J}\left(\mathcal{H}^{D}\right)$ is cyclic, so $\mathbf{S}$ has an SSIR. Let $\mathcal{F} \equiv\left\{F_{\alpha}\right\}$ be a Cauchy filter base on $\mathbf{S}$. The projection image $\mathcal{F}^{D} \equiv\left\{F_{\alpha}^{D} \equiv \operatorname{Proj}_{\mathcal{H}^{D}} F_{\alpha}\right\}$ for any $D \in \Omega$ gives a Cauchy filter base on $\mathbf{J}\left(\mathcal{H}^{D}\right)$. Conversely, for a filter base $\mathcal{F} \equiv\left\{F_{\alpha}\right\}_{\alpha \in \Gamma}$ on $\mathbf{J}(\Omega)$ to be Cauchy, it is enough that, for any $D$ in $\Omega, \mathcal{F}^{D}$ is Cauchy. Since on $\mathbf{J}(\Omega)$ the weak topology is equivalent to the strong topology, we can consider these Cauchy properties in the sense of strong topology on $\mathbf{J}(\Omega)$. 
For any $v \in \mathcal{H}^{D}$ for a fixed $D$, a Cauchy filter base $\left\{F_{\alpha}^{D} v\right\}_{\alpha \in \Gamma}$ converges to a vector $u(v)$ in the Hilbert space $\mathcal{H}^{D}$; that is, for any $J_{\alpha}^{D} \in F_{\alpha}^{D}$ and any $v \in \mathcal{H}^{D}$,

$$
\text { strong- } \lim _{\alpha} J_{\alpha}^{D} v=u(v) \text {. }
$$

Moreover, for any $a, b \in \mathbf{C}$,

$$
\lim _{\alpha} J_{\alpha}^{D}\left(a v_{1}+b v_{2}\right)=a u\left(v_{1}\right)+b u\left(v_{2}\right), \quad\|u(v)\|=\lim _{\alpha}\left\|J_{\alpha}^{D} v\right\|=\|v\| .
$$

Therefore, the map $\mathcal{H}^{D} \ni v \mapsto u(v) \in \mathcal{H}^{D}$ is linear and isometric. Thus there exists an isometric operator $B^{D}$ such that $u(v)=B^{D} v$.

\section{LEMMA 2.2}

Any Cauchy filter base on $\mathbf{J}(\Omega)=\prod_{D \in \Omega} \mathbf{J}\left(\mathcal{H}^{D}\right)$ converges to a $\mathbf{B} \equiv\left(B^{D}\right)_{D \in \Omega} \in$ $\mathbf{J}(\Omega)$, where the $B^{D}$ 's are isometric operators.

For a topological semigroup $S$, any filter base $\mathcal{F}$ on it is mapped to a filter base $\mathcal{F}_{J}$ in $S_{J}$. And if $\mathcal{F}$ is Cauchy, then $\mathcal{F}_{J}$ in $\mathbf{J}(\Omega)$ is also Cauchy.

\section{LEMMA 2.3}

$A$ Cauchy filter base $\mathcal{F}_{J}$ on a semigroup $S_{J}$ converges to an element $\mathbf{B} \equiv$ $\left(B^{D}\right)_{D \in \Omega}$ in $\mathbf{J}(\Omega)$.

\section{DEFINITION 2.3}

We say that a $T_{2}$-topological semigroup $S$ is of T-type if

(T-1) $S$ has an SSIR,

(T-2) $S$ is complete.

\section{Birepresentations of $S$}

A birepresentation $\mathbf{A} \equiv\left\{A^{D}\right\}_{D \in \Omega}$ of $S$ with an SSIR has analogous properties to a birepresentation in the case of groups. We argue similarly as in [4, Section 6$]$ and [5, Section 2].

Consider the contragradient representation $\bar{D}$ of $D$, consider the vector $\bar{v}$ in $\mathcal{H}^{\bar{D}}$ corresponding to $v$ in $\mathcal{H}^{D}$, and consider $\bar{A}$ the operator on $\mathcal{H}^{\bar{D}}$ corresponding to $A$ on $\mathcal{H}^{D}$. By the condition (Cd-4) of the definition of birepresentation,

$$
\overline{A^{D}}=A^{\bar{D}} \text {. }
$$

From this, by the same calculations as in [5, Lemma 2.1 and Corollary 2.1.1], we can obtain the following.

(1) $\left\langle A^{D \oplus \bar{D}}(u \oplus \bar{u}), v \oplus \bar{v}\right\rangle$ is real valued.

(2) Denote by $I \equiv\left\{\mathbf{C}, I_{g}, v_{0}\right\}$ the trivial representation of $S$, and put $D_{p} \equiv$ $I \oplus D \oplus \bar{D}$. Take vectors $w_{0} \in \mathcal{H}^{I}$ and $w \in \mathcal{H}^{D}$ such that $2^{1 / 2}\left\|w_{0}\right\|=2\|w\|=1$, and put $v_{p} \equiv w_{0} \oplus w \oplus \bar{w}$ in $\mathcal{H}^{I} \oplus \mathcal{H}^{D} \oplus \mathcal{H}^{\bar{D}}$. Then for any $g \in S$ the matrix 
element

$$
\left\langle T_{g}^{D_{p}} v_{p}, A^{D_{p}} v_{p}\right\rangle=\left\langle T_{g}^{D_{p}}\left(w_{0} \oplus w \oplus \bar{w}\right), A^{D_{p}}\left(w_{0} \oplus w \oplus \bar{w}\right)\right\rangle \geq 0 .
$$

By an argument analogous to [4, Corollary 1.2.2], we get the following.

\section{LEMMA 3.1}

Let $D$ and $D_{p}$ be as above. Then $1>\forall \varepsilon>0, \forall g_{0} \in S, \exists \delta>0$,

$$
F\left(D_{p}, \delta, g_{0}\right) \subset F\left(D, \varepsilon, g_{0}\right) .
$$

\section{Proof}

Put $\eta(g) \equiv\left\langle T_{g}^{D} v^{D}, T_{g_{0}}^{D} v^{D}\right\rangle$, and put $\eta_{p}(g) \equiv\left\langle T_{g}^{D_{p}} v^{D_{p}}, T_{g_{0}}^{D_{p}} v^{D_{p}}\right\rangle$. Then $1-\eta_{p}(g)=$ $2^{-1}(1-\Re \eta(g))$ and

$$
\begin{aligned}
\|1-\eta(g)\|^{2} & =(1-\Re \eta(g))^{2}+(\Im \eta(g))^{2} \\
& \leq(1-\Re \eta(g))^{2}+(1-\Re \eta(g))(1+\Re \eta(g)) \\
& \leq 3(1-\Re \eta(g))=6\left(1-\eta_{p}(g)\right) .
\end{aligned}
$$

This shows that if $6 \delta<\varepsilon^{2}$, then $F\left(D^{p}, \delta, g_{0}\right) \subset F\left(D, \varepsilon, g_{0}\right)$.

We get also the following result.

\section{COROLLARY 3.1.1}

If $\{D\}$ gives an SSIR of $S$, then $\left\{D_{p}\right\}$ is also an SSIR of $S$.

Take $D=\left\{\mathcal{H}^{D}, T_{g}^{D}, v^{D}\right\}$ a cyclic representation of $S$, and put $K^{D}(g) \equiv\left\langle T_{g}^{D} v^{D}\right.$, $\left.A^{D} v^{D}\right\rangle$.

\section{LEMMA 3.2}

Let $\mathbf{A} \equiv\left\{A^{D}\right\}_{D \in \Omega}$ be a birepresentation of $S$ with an SSIR. Then for any cyclic $D \equiv\left\{\mathcal{H}^{D}, T_{g}^{D}, v^{D}\right\}\left(\left\|v^{D}\right\|=1\right)$ in $\Omega$,

$$
\sup _{g \in S}\left|K^{D}(g)\right|=1 \text {. }
$$

Proof

The arguments are similar to the proof of [4, Lemma 2.2]. At first, obviously $\left|K^{D}(g)\right| \leq 1$. The relations for $\zeta^{D}(g)=\left\langle T_{g}^{D} v^{D}, u^{D}\right\rangle$,

$$
\begin{aligned}
\overline{\zeta^{D}(g)} & =\zeta^{\bar{D}}(g), \\
\zeta^{D_{1}}(g)+\zeta^{D_{2}}(g) & =\zeta^{D_{1} \oplus D_{2}}(g), \\
\zeta^{D_{1}}(g) \times \zeta^{D_{2}}(g) & =\zeta^{D_{1} \otimes D_{2}}(g),
\end{aligned}
$$

show that, when $D$ runs over $\Omega$ and $u, v$ run over any vectors in $\mathcal{H}^{D}$, the family $\mathfrak{F} \equiv\left\{\zeta^{D}(g)\right\}$ of matrix elements gives a $*$-algebra contained in the $*$-algebra $\mathcal{C}^{b}(S)$ of all bounded continuous functions on $S$ with the norm $\left\|\zeta^{D}\right\| \equiv \sup _{g \in S}\left|\zeta^{D}(g)\right|$. 
The completion $\mathfrak{F}^{C}$ of $\mathfrak{F}$ with respect to this norm is a $C^{*}$-algebra of continuous functions on $S$.

By Gelfand's representation theorem, $\mathfrak{F}^{C}$ is isomorphic to the space $\mathcal{C}^{b}(X)$ of all bounded continuous functions on a locally compact space $X$ under the correspondence $\mathfrak{F}^{C} \ni f \mapsto f^{\sim} \in \mathcal{C}^{b}(X)$. A point $x$ of $X$ is a homomorphic map such that

$$
\begin{aligned}
\psi^{x}: \mathcal{C}^{b}(X) & \rightarrow \mathbf{C}, \\
\psi^{x}(\varphi) & \equiv \varphi(x) \quad\left(\varphi \in \mathcal{C}^{b}(X)\right) .
\end{aligned}
$$

For any element $g$ in $S$ and $f$ in $\mathfrak{F}^{C}$,

$$
f \mapsto f(g)
$$

gives a homomorphic map from $\mathfrak{F}^{C}$ to $\mathbf{C}$. So there exists a unique element $x_{g}$ in $X$ as

$$
f(g)=f^{\sim}\left(x_{g}\right) .
$$

The existence of an SSIR ensures that the map $g \mapsto x_{g}$ is one-to-one. So by this map, $S$ is embedded into $X$. But $\mathcal{C}^{b}(X)$ is given as the space of $\left\{f^{\sim} \mid f \in \mathfrak{F}^{C}\right\}$ and $\mathfrak{F}^{C} \subset \mathcal{C}^{b}(S)$. This implies that the image of $S$ is dense in $X$. So for any $x \in X$, $\delta>0$, and $f^{\sim} \in \mathfrak{F}^{C}$, there exists $g_{0} \in S$ such that

$$
\left|f^{\sim}\left(g_{0}\right)-f^{\sim}(x)\right|<\delta .
$$

For a given birepresentation $\mathbf{A} \equiv\left\{A^{D}\right\}_{D \in \Omega}$, consider the map

$$
\zeta^{D}(g)=\left\langle T_{g}^{D} v^{D}, u^{D}\right\rangle \mapsto\left\langle A^{D} v^{D}, u^{D}\right\rangle \equiv \theta_{\mathbf{A}}\left(\zeta^{D}\right) .
$$

By considerations analogous to those in (3.5), (3.6), and (3.7), we get that

$$
\begin{aligned}
\overline{\theta_{\mathbf{A}}\left(\zeta^{D}\right)} & =\left\langle\overline{A^{D} v^{D}, u^{D}}\right\rangle=\left\langle\overline{A^{D}} v^{\bar{D}}, u^{\bar{D}}\right\rangle=\theta_{\mathbf{A}}\left(\zeta^{\bar{D}}\right), \\
\theta_{\mathbf{A}}\left(\zeta^{D_{1}}\right)+\theta_{\mathbf{A}}\left(\zeta^{D_{2}}\right) & =\left\langle A^{D_{1}} v^{D_{1}}, u^{D_{1}}\right\rangle+\left\langle A^{D_{2}} v^{D_{2}}, u^{D_{2}}\right\rangle \\
& =\left\langle\left(A^{D_{1}} v^{D_{1}} \oplus A^{D_{2}} v^{D_{2}}\right),\left(u^{D_{1}} \oplus u^{D_{2}}\right)\right\rangle=\theta_{\mathbf{A}}\left(\zeta^{D_{1} \oplus D_{2}}\right), \\
\theta_{\mathbf{A}}\left(\zeta^{D_{1}}\right) \times \theta_{\mathbf{A}}\left(\zeta^{D_{2}}\right) & =\left\langle A^{D_{1}} v^{D_{1}}, u^{D_{1}}\right\rangle \times\left\langle A^{D_{2}} v^{D_{2}}, u^{D_{2}}\right\rangle \\
& =\left\langle\left(A^{D_{1}} v^{D_{1}} \otimes A^{D_{2}} v^{D_{2}}\right),\left(u^{D_{1}} \otimes u^{D_{2}}\right)\right\rangle=\theta_{\mathbf{A}}\left(\zeta^{D_{1} \otimes D_{2}}\right) .
\end{aligned}
$$

Consider the case in which $\sum_{j}\left\langle T_{g}^{D_{j}} v^{D_{j}}, u^{D_{j}}\right\rangle \equiv 0$ as a function on $S$ for some countable set $\left\{D_{j}\right\} \subset \Omega$ and $\left\{v^{D_{j}}, u^{D_{j}} \in \mathcal{H}^{D_{j}}\right\}$ such that $\sum_{j}\left\|v^{D_{j}}\right\|^{2}$, $\sum_{j}\left\|u^{D_{j}}\right\|^{2}<\infty$.

Put $D \equiv \sum_{j}^{\oplus} D_{j}$, put $v^{D} \equiv \sum_{j}^{\oplus} v^{D_{j}}$, and put $u^{D} \equiv \sum_{j}^{\oplus} u^{D_{j}}$. Then for any $g \in S,\left\langle T_{g}^{D} v^{D}, u^{D}\right\rangle \equiv 0$.

The condition (Cd-2) of the definition of birepresentation $\mathbf{A} \equiv\left\{A^{D}\right\}_{D \in \Omega}$ shows that the operator $A^{D}$ keeps the invariant subspace $H$ spanned by $\left\{T_{g}^{D} v^{D}(g \in G)\right\}$, that is, $A^{D} H \perp u^{D}$, and

$$
0=\left\langle A^{D} v^{D}, u^{D}\right\rangle=\sum_{j}\left\langle A^{D_{j}} v^{D_{j}}, u^{D_{j}}\right\rangle .
$$


Therefore, map (3.12) generates a $*$-algebra homomorphism

$$
f^{\sim}(g) \mapsto \theta_{\mathbf{A}}\left(f^{\sim}\right) \equiv f^{\sim}\left(x_{\mathbf{A}}\right)
$$

of the space $\mathfrak{F}$ and of $\mathfrak{F}^{C}$ to $\mathbf{C}$; that is, it gives an element $x_{\mathbf{A}} \in X$ by the above equation.

Put $f^{\sim}(g) \equiv\left\langle T_{g}^{D} v^{D}, A^{D} v^{D}\right\rangle$, and apply (3.11). We obtain that

$$
\begin{aligned}
\left|f^{\sim}\left(g_{0}\right)-f^{\sim}\left(x_{\mathbf{A}}\right)\right| & =\left|\left\langle T_{g_{0}}^{D} v^{D}, A^{D} v^{D}\right\rangle-\left\langle A^{D} v^{D}, A^{D} v^{D}\right\rangle\right| \\
& =\left|\left\langle T_{g_{0}}^{D} v^{D}, A^{D} v^{D}\right\rangle-1\right|=\left|1-K^{D}\left(g_{0}\right)\right|<\delta .
\end{aligned}
$$

This proves (3.4).

Let $\Omega_{+}$be the set of all cyclic representations $D=\left(\mathcal{H}^{D}, T_{g}^{D}, v^{D}\right)\left(\left\|v^{D}\right\|=1\right)$ satisfying

$$
K^{D}(g)=\left\langle T_{g}^{D} v^{D}, A^{D} v^{D}\right\rangle \geq 0 \quad(g \in S) .
$$

Then, by Lemma 3.2, for $D \in \Omega_{+}$,

$$
\inf _{g \in S}\left(1-K^{D_{p}}(g)\right)=0
$$

And $\Omega_{+}$contains cyclic representations of type $\left(D_{p}\right)$. Put

$$
\begin{aligned}
E(D, \varepsilon) & \equiv\left\{g \mid 1-K^{D}(g)<\varepsilon\right\}, \\
\mathrm{Z} & \equiv\{E(D, \varepsilon)\}_{D \in \Omega_{+}, \varepsilon>0} .
\end{aligned}
$$

\section{LEMMA 3.3}

For a birepresentation $\mathbf{A}=\left(A^{D}\right)_{D \in \Omega}$ of $S$ with an SSIR, Z gives a Cauchy filter base on $S$.

Proof

Lemma 3.2 shows that $E(D, \varepsilon)$ is not empty and

$$
\varepsilon_{1}>\varepsilon_{2} \Rightarrow E\left(D, \varepsilon_{1}\right) \supseteq E\left(D, \varepsilon_{2}\right) .
$$

Let $D^{0} \equiv\left(D^{1} \otimes D^{2}\right)$ (the cyclic part in $D^{1} \otimes D^{2}$ ) with $D^{1}, D^{2} \in \Omega_{+}$. Then

$$
\begin{aligned}
1-K^{D^{0}}(g) & \geq 1-K^{D^{1}}(g), \quad 1-K^{D^{0}}(g) \geq 1-K^{D^{2}}(g), \\
E\left(D^{1}, \varepsilon\right) \cap E\left(D^{2}, \varepsilon\right) & \supseteq E\left(D^{0}, \varepsilon\right) \neq \phi .
\end{aligned}
$$

So $\mathrm{Z}$ is a filter base.

Analogous calculations to $[4,(7.8)]$ show that $1-K^{D}(g)<\varepsilon$ leads to

$$
\left\|A^{D} v^{D}-T_{g}^{D} v^{D}\right\| \leq(2 \varepsilon)^{1 / 2} .
$$

Hence, for any $g, h \in E(D, \varepsilon)$,

$$
\left\|T_{g}^{D} v^{D}-T_{h}^{D} v^{D}\right\| \leq 2(2 \varepsilon)^{1 / 2} .
$$


For an arbitrary given entourage $W\left(D, 2(2 \varepsilon)^{1 / 2}\right)$ in $S \times S$, if we take the above $E(D, \varepsilon)$, then

$$
\forall g, h \in E(D, \varepsilon), \quad(g, h) \in W\left(D, 2(2 \varepsilon)^{1 / 2}\right)
$$

that is, $\mathrm{Z}$ is Cauchy.

\section{Proof of Tannaka-type duality theorem for T-type semigroup}

\section{THEOREM 4.1}

For a T-type semigroup $S$, the Tannaka-type duality theorem is valid.

Proof

For any given birepresentation $\mathbf{A} \equiv\left\{A^{D}\right\}$, we show that there exists a unique $g$ in $S$ such that

$$
\left\{A^{D}\right\}=\left\{T_{g}^{D}\right\} .
$$

The T-type semigroup $S$ is complete by definition, and $\mathrm{Z} \equiv\{E(D$, $\varepsilon)\}_{D \in \Omega_{+}, \varepsilon>0}$ is a Cauchy filter base. So there exists a limit point $g_{\mathbf{A}}$ and

$$
\bigcap_{(D, \varepsilon)} \overline{E(D, \varepsilon)}=\left\{g_{\mathbf{A}}\right\} .
$$

Therefore, $1=K^{D}\left(g_{\mathbf{A}}\right)=\left\langle T_{g_{\mathbf{A}}}^{D} v^{D}, A^{D} v^{D}\right\rangle$, that is,

$$
\forall D \in \Omega_{+}, \quad A^{D} v^{D}=T_{g_{\mathrm{A}}}^{D} v^{D} .
$$

For a general cyclic representation $D$, consider $\left(D_{p}\right) \in \Omega_{+}$as in Section 3; then we get from $A^{D_{p}} v_{p}=T_{g_{\mathrm{A}}}^{D_{p}} v_{p}$ that

$$
I w_{0} \oplus A^{D} w \oplus A^{\bar{D}} \bar{w}=I w_{0} \oplus T_{g_{\mathbf{A}}}^{D} w \oplus T_{g_{\mathbf{A}}}^{\bar{w}} \bar{w} .
$$

So we get, for any $D$ in $\Omega$, that $A^{D} w=T_{g_{\mathbf{A}}}^{D} w$. This concludes the proof.

\section{Converse of Theorem 4.1}

\section{LEMMA 5.1}

For a $T_{2}$-topological semigroup $S$, if a weak Tannaka-type duality theorem holds, then $S$ has an SSIR.

Proof

By Lemma 2.1, the inverse map of (2.3) from $S_{J}$ to $S$ must be continuous. A fundamental system of neighborhoods $V$ of $\left(T_{g_{0}}^{D}\right)$ in $S_{J}$ is given as the collection of

$$
V_{1} \equiv \bigcap_{1 \leq j \leq n}\left\{\mathbf{T}_{g}=\left(T_{g}^{D}\right)_{D \in \Omega} \mid\left\|T_{g}^{D_{j}} v_{j}-T_{g_{0}}^{D_{j}} v_{j}\right\|^{2}<\varepsilon_{j}\right\}
$$

for a finite set $\left\{\left(D_{j}, v_{j}, \varepsilon_{j}\right)\right\}$, where $D_{j} \in \Omega, v_{j} \in \mathcal{H}^{D_{j}}\left(\left\|v_{j}\right\|=1\right), \varepsilon_{j}>0(j=$ $1,2, \ldots, n)$. 
Consider the representation $D_{0} \equiv \sum_{j}^{\oplus} D_{j}$, consider $v_{0}=n^{-(1 / 2)} \sum_{j}^{\oplus} v_{j}$, and consider $\varepsilon_{0}=\min _{j} \varepsilon_{j}$. Then

$$
V_{1} \supseteq V_{2}\left(\varepsilon_{0}\right) \equiv\left\{\mathbf{T}_{g}=\left(T_{g}^{D}\right)_{D \in \Omega} \mid\left\|v_{0}-T_{g}^{D_{0}} v_{0}\right\|^{2}<\varepsilon_{0}\right\} .
$$

The evaluation

$$
\begin{aligned}
\left\|T_{g}^{D_{0}} v_{0}-T_{g_{0}}^{D_{0}} v_{0}\right\|^{2} & =2\left(1-\Re\left(\left\langle T_{g}^{D_{0}} v_{0}, T_{g_{0}}^{D_{0}} v_{0}\right\rangle\right)\right) \\
& \leq 2\left|1-\left\langle T_{g}^{D_{0}} v_{0}, T_{g_{0}}^{D_{0}} v_{0}\right\rangle\right|
\end{aligned}
$$

shows that if we take $\delta<2^{-1} \varepsilon_{0}$, then

$$
V_{2}\left(\varepsilon_{0}\right) \supset V_{\delta} \equiv\left\{\mathbf{T}_{g}=\left(T_{g}^{D}\right)_{D \in \Omega}|| 1-\left\langle T_{g}^{D_{0}} v_{0}, T_{g_{0}}^{D_{0}} v_{0}\right\rangle \mid<\delta\right\} .
$$

For any neighborhood $V$ of $g_{0}$ in $G$, there exist $V, V_{1}, V_{2}\left(\epsilon_{0}\right)$, and $V_{\delta}$ such that

$$
V \supseteq V_{1} \supseteq V_{2}\left(\epsilon_{0}\right) \supseteq V_{\delta} .
$$

This shows the separating condition of the existence of an SSIR in Definition 2.3.

\section{LEMMA 5.2}

For a $T_{2}$-topological semigroup $S$, if the Tannaka-type duality theorem holds, then $S$ must be complete.

Proof

For any Cauchy filter base $\mathcal{F}$ on $S$, its image $\mathcal{F}_{J}$ in $S_{J} \subset \mathbf{J}(\Omega)$ is also Cauchy. And by Lemma 2.2, it converges to an isometric operator field $\mathbf{A}_{0} \equiv\left\{A_{0}^{D}\right\}$. We can easily confirm that $\mathbf{A}_{0}$ gives a birepresentation, that is,

$$
\mathbf{A}_{0} \in S_{J}
$$

From the assumption that the Tannaka-type duality theorem is valid, $\mathcal{F}$ must converge to a point in $S$, the inverse image of $\mathbf{A}_{0}$.

Summarizing the results of Lemmas 5.1 and 5.2, we have the following.

\section{THEOREM 5.1}

For a $T_{2}$-topological semigroup $S$, if the Tannaka-type duality theorem holds, then $S$ must be a T-type semigroup.

\section{Main theorem and example}

Summarizing Theorems 4.1 and 5.1, we obtain the following.

\section{MAIN THEOREM}

Let $S$ be a $T_{2}$-topological semigroup. For $S$, the Tannaka-type duality theorem holds if and only if $S$ is a T-type semigroup. 


\section{EXAMPLE 1}

Let $\mathcal{H}$ be a Hilbert space of infinite dimension, and let $S \equiv \mathbf{J}(\mathcal{H})$ be the semigroup of all isometric operators on $\mathcal{H}$ with the weak (=strong) topology of operator space.

\section{LEMMA 6.1}

The semigroup $S \equiv \mathbf{J}(\mathcal{H})$ is a $T_{2}$-topological semigroup and has an SSIR.

Proof

As we showed in Section 2, $S$ is a complete $T_{2}$-topological semigroup. Consider the identical representation $D_{0} \equiv\left\{\mathcal{H}, T_{J}\right\}$,

$$
S \ni J \mapsto T_{J}(\equiv J) \in \mathbf{J}(\mathcal{H}) .
$$

The family of all cyclic subrepresentations gives an SSIR of $S$.

Lemma 2.3 claims that $S$ is complete, so $S$ is of T-type. And the Tannaka-type duality theorem holds for $S$.

\section{Extension to a topological group}

\section{LEMMA 7.1}

Let $S_{1}$ be a semigroup of isometric operators on a Hilbert space $\mathcal{H}$ with the weak (=strong) topology of operator space. Then $S_{1}$ is a topological semigroup. Moreover, if $S_{1}$ is a group of unitary operators, then $S_{1}$ is a topological group.

Proof

The relation for $T_{1}, T_{2} \in S_{1}$,

$$
\left\|T_{1} T_{2} v-v\right\| \leq\left\|T_{1} T_{2} v-T_{1} v\right\|+\left\|T_{1} v-v\right\|=\left\|T_{2} v-v\right\|+\left\|T_{1} v-v\right\|,
$$

shows the continuity of multiplication on $S_{1}$ with respect to the strong topology.

We denote by $\mathbf{U}(\mathcal{H})$ the space of all unitary operators on a Hilbert space $\mathcal{H}$, and introduce the weak topologies on it. Let $\Omega_{0} \equiv\left\{D=\left(\mathcal{H}^{D}, T_{g}^{D}\right)\right\}$ be a set of unitary representations of some topological semigroup $S$. Construct $\mathbf{U}\left(\Omega_{0}\right) \equiv$ $\prod_{D \in \Omega_{0}} \mathbf{U}\left(\mathcal{H}^{D}\right)$ with the natural product topology.

\section{COROLLARY 7.1.1}

We have that $\mathbf{U}\left(\Omega_{0}\right)$ is a topological group.

Proof

As the product of topological groups $\mathbf{U}\left(\mathcal{H}^{D}\right), \mathbf{U}\left(\Omega_{0}\right)$ is a topological group. 
LEMMA 7.2

Any subgroup with the relative topology of a topological group is a topological group.

Proof

The proof is obvious.

PROPOSITION 7.1

Let $S$ be a T-type $T_{2}$-topological semigroup. If $S$ has an SSIR $\Omega_{1} \equiv\left\{D_{\alpha} \equiv\right.$ $\left\{\mathcal{H}^{D_{\alpha}}, T_{g}^{D_{\alpha}}, v^{D_{\alpha}}\right\} \mid v^{D_{\alpha}}$ is a normalized cyclic vector, $\left.\alpha \in A\right\}$, all elements $D_{\alpha}$ of which are unitary representations, then there exists a topological group $G$ which contains $S$ as a topological subsemigroup.

Proof

Write $\Omega_{0}$ as the set of all unitary representations of $S$. From the assumption, $\Omega_{0}$ gives an SSIR of $S$.

For a given birepresentation $\mathbf{T}_{g} \equiv\left\{T_{g}^{D}\right\}_{D \in \Omega}$ of $S$, we consider the operator field $\left(\mathbf{T}_{g}\right)^{-1} \equiv\left\{\left(T_{g}^{D}\right)^{-1}\right\}_{D \in \Omega_{0}}$ on $\Omega_{0}$ and take the group $G$ generated by the family $\left\{\mathbf{T}_{g},\left(\mathbf{T}_{g}\right)^{-1} \mid g \in S\right\}$. Then, $G$ is in $\mathbf{U}\left(\Omega_{0}\right)$. So the above lemmata show that $G$ is a topological group with the topology in $\mathbf{U}\left(\Omega_{0}\right)$, containing $S$.

But $\Omega_{0}$ gives an SSIR of $S$. So the topology of $S$ just coincides with the restricted one of $G$.

\section{COROLLARY}

Let $S$ be a T-type $T_{2}$-topological semigroup. If all representations of $S$ are unitary representations, then $S$ must be a topological group.

Proof

In this case, $\Omega=\Omega_{0}$. So $G$ is the set of all birepresentations of $S$. Therefore, the Tannaka-type duality theorem claims that $S=G$.

\section{EXAMPLE 2}

Consider the case where $S$ is the additive semigroup $\mathbf{R}_{+}$of all nonnegative real numbers. Let $\mu$ be the ordinary Lebesgue measure on $S$, and let $\mathcal{H}$ be the space $L^{2}(S, \mu)$.

The right translation operator $T_{g_{0}}$ defined by

$$
\begin{aligned}
T_{g_{0}} f(g) & =0 \quad\left(g \notin g_{0}+S\right) \\
& =f\left(g_{1}\right) \quad\left(g=g_{0}+g_{1} \in g_{0}+S\right)
\end{aligned}
$$

gives an isometric operator on $\mathcal{H}$. Let $\mathcal{R} \equiv\left\{\mathcal{H}, T_{g}\right\}$ define a nonunitary but isometric representation of $S$. It is easy to see that $\{\mathcal{R}\}$ is an SSIR, and $S$ is complete. Thus, $S$ is a T-type semigroup, and the Tannaka-type duality theorem is valid. 
Now we consider the space $\mathcal{H}^{0} \equiv L^{2}\left(\mathbf{R}, \mu^{0}\right)$ (where $\mu^{0}$ is the ordinary Lebesgue measure on $\mathbf{R}$ ) and the representation $\mathcal{R}^{0} \equiv\left(\mathcal{H}^{0}, T_{g}^{0}\right)$ (where $T_{g}^{0}$ is the translation by $g$ ). Note that $\mathcal{R}^{0}$ is a unitary representation. And $S$ is embedded in the additive group of real numbers $\mathbf{R}$ which has a representation $\mathcal{R}^{0}$ on $\mathcal{H}^{0}$, extending $\mathcal{R}$. Thus, if we treat only unitary representations, then we get the whole additive group $\mathbf{R}$ as the set of birepresentations of $S$.

\section{References}

[1] N. Bourbaki, Topologie générale, chapitre 2, Hermann, Paris, 1951.

[2] N. Tatsuuma, A duality theorem for locally compact groups, J. Math. Kyoto Univ. 6 (1967), 187-293. MR 0217222.

[3] - "Duality theorem for inductive limit group of direct product type" in Representation Theory and Analysis on Homogeneous Spaces, RIMS Kôkyûroku Bessatsu B7, Res. Inst. Math. Sci. (RIMS), Kyoto, 2008, 13-23. MR 2449443.

[4] _ Duality theorem for inductive limit groups, Kyoto J. Math. 54 (2014), 51-73. MR 3178546. DOI 10.1215/21562261-2400274.

[5] - Duality theorems and topological structure of groups, Kyoto J. Math. 54 (2014), 75-101. MR 3178547. DOI 10.1215/21562261-2400283.

[6] K. Yosida, Functional Analysis, Springer, Berlin, 1971.

Nishinomiya City, Matsuoi-chô 10-8, Japan 